Onkologe 2011 · 17:362-363

DOI 10.1007/s00761-011-2048-2

Online publiziert: 20. April 2011

(c) Springer-Verlag 2011

\author{
K. Höffken ${ }^{1}$ · P.M. Schlag ${ }^{2}$ - M. Bamberg ${ }^{3}$ \\ ${ }^{1}$ Universitätsklinikum Jena \\ ${ }^{2}$ Charité Comprehensive Cancer Center, Charité - \\ Universitätsmedizin Berlin, Campus Mitte, Berlin \\ ${ }^{3}$ Abteilung für Strahlentherapie, Radiologische Universitätsklinik, Tübingen
}

\title{
E pluribus unum ...
}

risierter Operationsinstrumentarien mit teilautonomen und mechatronischen $\mathrm{Na}-$ vigationshilfen wesentlich dazu beigetragen haben, tumorchirurgische Eingriffe effizienter, sicherer und weniger traumatisierend durchzuführen.

Wer die Entwicklung der verfügbaren Methoden und Substanzen zur Systemtherapie maligner Erkrankungen miterlebt hat, weiß, welche Morbidität und Effektivität mit der geringen Spezifität und der zunächst zu akzeptierenden Toxizität dieser Verfahren verbunden war. Welch ein Wandel ist hier zu konstatieren und welche Veränderungen zum Positiven sind beispielsweise durch neuartige, maßgenau auf Tumor und Patient zugeschnittene „personalisierte“ Behandlungsverfahren oder Pharmakotherapie mittels Nanomedizin noch zu erwarten!

Diese Themen, deren praktischer Anwendungshorizont teilweise noch weit in der Zukunft liegt, werden in den vorliegenden Beiträgen mit hoher Expertise beschrieben. Fehlen darf weder der Blick auf Nutzen und Kosten noch auf die schnellere Umsetzung neuer Erkenntnisse durch eine adäquate Weitergabe an die praktizierenden Ärzte.

Herausgeber und Verlagsredaktion haben sich zum Ziel gesetzt, auch zukünftig wieder wichtige aktuelle Themen durch Auswahl von zukunftsweisenden Beiträgen aus den vielen anderen Fachzeitschriften des Springer-Verlags zu einem Leitthema zusammen zu fassen und unserer Leserschaft die Möglichkeit zu geben, auf diese Weise ihren Kenntnisstand zu erweitern.

Das vorliegende Schwerpunktheft über neue Techniken in Diagnostik und Therapie macht einen Anfang.

Die Herausgeber
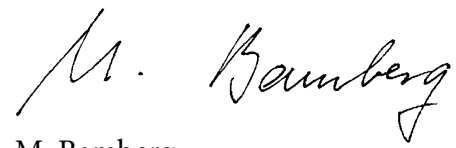

M. Bamberg

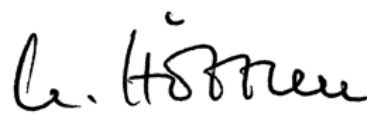

K. Höffken

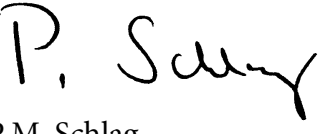

P.M. Schlag

\section{Korrespondenzadresse \\ Prof. Dr. K. Höffken \\ Universitätsklinikum Jena \\ Bachstraße 18, 07743 Jena \\ Hoeffken.Editor@med.uni-jena.de}


Hier steht eine Anzeige.

黛 Springer 